\title{
X-Linked Periventricular Nodular Heterotopia a Rare Cause of Headache and Right Sided Hemiparesis in a 7-Year-Old Girl
}

\author{
Christine Loftis, BS*, Aleksandr Chernyy, MD and Randy Williamson, MD \\ University of Texas Rio Grande Valley School of Medicine, USA
}

\section{Introduction}

X-linked Periventricular Nodular Heterotopia (X-linked PVNH) is a neuronal migration disorder that occurs because nerve cells fail to migrate during fetal development. The disorder is caused by a defect in the FLNA gene which is responsible for making the protein filamin $A$ which is responsible for cellular structure [1]. Without filamin A, neurons begin to build up near the brain's ventricles and can ultimately result in seizures, intellectual disability and are associated with an increased risk of cardiovascular and cerebrovascular attacks [2]. Since X-linked PVNH is an $X$-linked dominant disorder, the disease typically affects females as the condition is lethal in males [3]. Most patients present with new onset seizures during adolescence, but in rare cases, as we will present, patients can present with stroke-like symptoms.

\section{Case Report}

A 7-year-old girl with a past medical history of recurrent headaches, ADHD and learning deficits presented for a two-week history of HA and two-day history of transient right sided weakness.

The patient's mother said that the patient had been complaining of severe headaches for 2 weeks. She was evaluated at an outside hospital where she had several diagnostic studies performed including a head CT, CXR, influenza testing, and RSV. She noted that she was informed that the tests were negative with the exception of the head CT which showed "white spots". The patient was discharged from that facility and was instructed to follow-up with a pediatric neurologist on an outpatient basis.

Following discharge, the patient's headaches intensified, and she began complaining of right upper and lower extremity weakness. The symptoms were transient in nature, however, when the patient was unable to walk, the patient's mother decided to bring her to our facility. The patient reported associated vomiting, but there was no recent fever, chills, change in speech, seizure like activity, loss of consciousness, change in vision, or any other symptoms.

The patient was found to be hypertensive to $139 / 59$ on admission. Physical examination was significant for a $2 / 6$ diastolic murmur heard best at the right second intercostal space but was negative for any focal neurological deficits. An echocardiogram was performed which showed significant aortic insufficiency, mild dilatation of the ascending aorta, and a PFO with left to right shunting. CT angiogram and CXR were negative. CT head and MRA were without any acute changes. MRI showed bilateral subependymal nodules which were isointense to gray matter which are findings consistent with X-linked PVNH. Based on clinical and MRI findings, patient was discharged with a working diagnosis of X-linked PVNH.

\section{Conclusion}

X-linked PVNH is a rare disorder that currently has no cure. Early diagnosis is imperative as interventions are geared to decreasing long term sequels from the disease such as cerebrovascular accidents or aortic or carotid dissection. The patient was treated symptomatically with ASA. She was discharged with referrals to a geneticist for confirmatory testing and pediatric cardiologist for the management of her aortic insufficiency and PFO.

\section{References}

1. Lange Max, Kasper B, Bohring A, et al. (2015) 47 Patients with FLNA associated periventricular nodular heterotopia. Orphanet J Rare Dis 10: 134.

2. Naviglio Samuele, Bruno I, Zanus C, et al. (2015) A brain and heart connection: X-linked periventricular heterotopia. J Pediatr 166: 776.

3. Fergelot Patricia, Isabelle Coupry, Caroline Roorycka, et al. (2012) Atypical male and female presentations of FLNA-related periventricular nodular heterotopia. European Journal of Medical Genetics 55: 313-318.

*Corresponding author: Christine Loftis, BS, MD Candidate, University of Texas Rio Grande Valley School of Medicine, USA

Accepted: April 18, 2020

Published online: April 20, 2020

Citation: Loftis C, Chernyy A, Williamson R (2020) X-Linked Periventricular Nodular Heterotopia a Rare Cause of Headache and Right Sided Hemiparesis in a 7-Year-Old Girl. J Pediatr Neurol Neurosci 4(1):54 